\title{
Diffuse Laminar Endocervical Hyperplasia
}

National Cancer Institute

\section{Source}

National Cancer Institute. Diffuse Laminar Endocervical Hyperplasia. NCI Thesaurus.

Code C127933.

A benign band-like proliferation of tightly-packed, small to medium sized endocervical glands below the surface of the endocervical canal. 